\title{
Editorial
}

\section{Spectroscopy in Materials Chemistry}

\author{
Tifeng Jiao, ${ }^{1}$ Nikša Krstulović, ${ }^{2}$ Bing Wu, ${ }^{3}$ Xinqing Chen, ${ }^{4}$ and Qingrui Zhang ${ }^{5}$ \\ ${ }^{1}$ School of Environmental and Chemical Engineering, Yanshan University, Qinhuangdao 066004, China \\ ${ }^{2}$ Institute of Physics, Bijenička 46, 10000 Zagreb, Croatia \\ ${ }^{3}$ Bodega Marine Laboratory, University of California, Davis, 2099 Westside Road, Bodega Bay, CA 94923, USA \\ ${ }^{4}$ Department of Chemical and Biomolecular Engineering, Hong Kong University of Science and Technology, Clear Water Bay, \\ Kowloon, Hong Kong \\ ${ }^{5}$ Department of Environmental Engineering, Yanshan University, Qinhuangdao 066004, China
}

Correspondence should be addressed to Tifeng Jiao; tfiao@ysu.edu.cn

Received 26 January 2015; Accepted 26 January 2015

Copyright (C) 2015 Tifeng Jiao et al. This is an open access article distributed under the Creative Commons Attribution License, which permits unrestricted use, distribution, and reproduction in any medium, provided the original work is properly cited.

Today, as nanotech continues to develop, materials chemistry is one of the key fields covering topics such as porous materials, metal-organic-framework materials, photocatalysts, magnetic materials, nanobiomaterials, and energy materials for fuel cells and batteries. The development of materials chemistry has made important progress through use of spectroscopic methods, such as UV-VIS-IR, OES, optical absorption spectroscopy, LIBS, XRD, vibrational spectroscopy, Raman/SERS/SERRS, photoluminescence, and photospectrometry/plasmonics, which are often supported by SEM, TEM, and AFM analyses to form the foundations of nanoscience and nanotechnology in recent decades. In addition, many scientists investigate also some special reaction mechanisms using spectroscopic tools.

This special issue addresses the research studies on the spectroscopy applications in materials chemistry. For example, W. Wang et al. reported the $\mathrm{CO}_{2}$ absorption by salinealkali soils in arid and semiarid ecosystems and developed the preliminary theory and methodology for the quantitative analysis. A. Li et al. investigated gas chromatography resonance-enhanced multiphoton ionization time-of-flight mass spectrometry (GC/REMPI-TOFMS) using a nanosecond laser to analyze the 16 polycyclic aromatic hydrocarbons (PAHs). G. Zhao et al. prepared fluorine-containing hydrophobic mesoporous material (MFS) with high surface area successfully synthesized with hydrothermal synthesis method by using a perfluorinated surfactant template. J. Liu et al. reported the preparation of $\mathrm{P}(\mathrm{AN}-\mathrm{VAc}-\mathrm{PMMT})$ nanocomposites using in situ emulsion polymerization and further confirmed it by FTIR. H. Guo et al. investigated the gelation behaviors of binary organogels composed of azobenzene amino derivatives and fatty acids with different alkyl chains in various organic solvents. L. K. Gao et al. reported the molecular dynamics simulations for the model diameter gradually decreasing the deformation mode of $\mathrm{ZrCu}$ metallic glass from highly localized shear band formation to homogeneous deformation with obvious transition. A. Wang et al. investigated the synthesis and surface modification of CdTe quantum dots (QDs) and the application in the rice growth. F. Jin reported the determination of stavudine in human plasma by LC-MS/MS method applied to the pharmaceutical formulations bioequivalence study. B. Sun et al. investigated the constrained optimization method employed to calculate the colorant values of the multispectral images. $\mathrm{H}$. Li et al. reported the utilization of infrared spectroscopy to examine the bone charcoal and the aluminum-impregnated bone charcoal. D. Yang et al. investigated the analysis for the oil content of 29 rapeseed samples based on near infrared spectral data with different wavelengths by an artificial neural network (ANN) method. L. Zhang and B. Qin reported a new technique named foamed gel developed to prevent coal mine fire efficiently. G. Zhao et al. reported the synthesis of a high surface area, hydrophobic mesoporous material (MFS) by a hydrothermal synthesis method using a perfluorinated surfactant, SURFLON S-386, as the single template. J. Lin et al. reported the preparation of zinc substituted magnesium 
ferrite powders by a sol-gel autocombustion method. J. Liu et al. investigated the heat-activated persulfate oxidative treatment of chlorinated organic solvents containing chlorinated ethenes and ethanes in soil with different persulfate dosages and temperatures. Y. Hu et al. reported the preparation of novel polyurethane microspheres containing curcumin (CurPUMs) using carboxymethyl cellulose sodium to improve the bioavailability and prolong the retention time of curcumin. In addition, $\mathrm{Y}$. Hu et al. also investigated the gelation behaviors of binary organogels composed of azobenzene amino derivatives and alkyloxybenzoic acids with different lengths of alkyl chains in various organic solvents.

\section{Acknowledgments}

Finally, the guest editors would like to express sincere appreciation to all the authors for their contributions. Moreover, thanks are extended to all reviewers for their time enhancing the quality of these papers.

Tifeng Jiao Nikša Krstulović Bing $W u$ Xinqing Chen Qingrui Zhang 

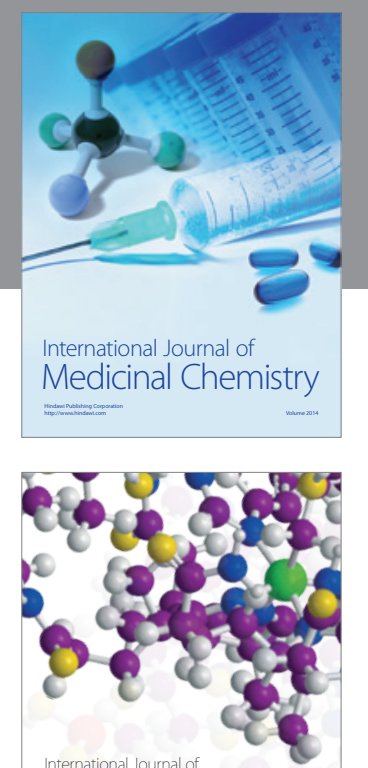

\section{Carbohydrate} Chemistry

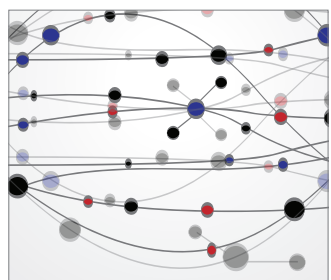

The Scientific World Journal
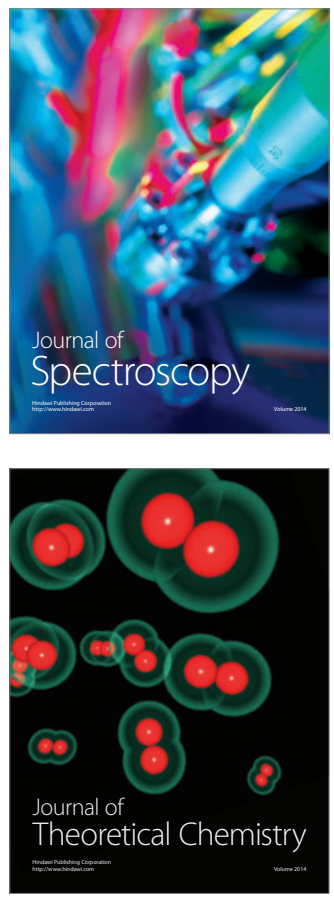
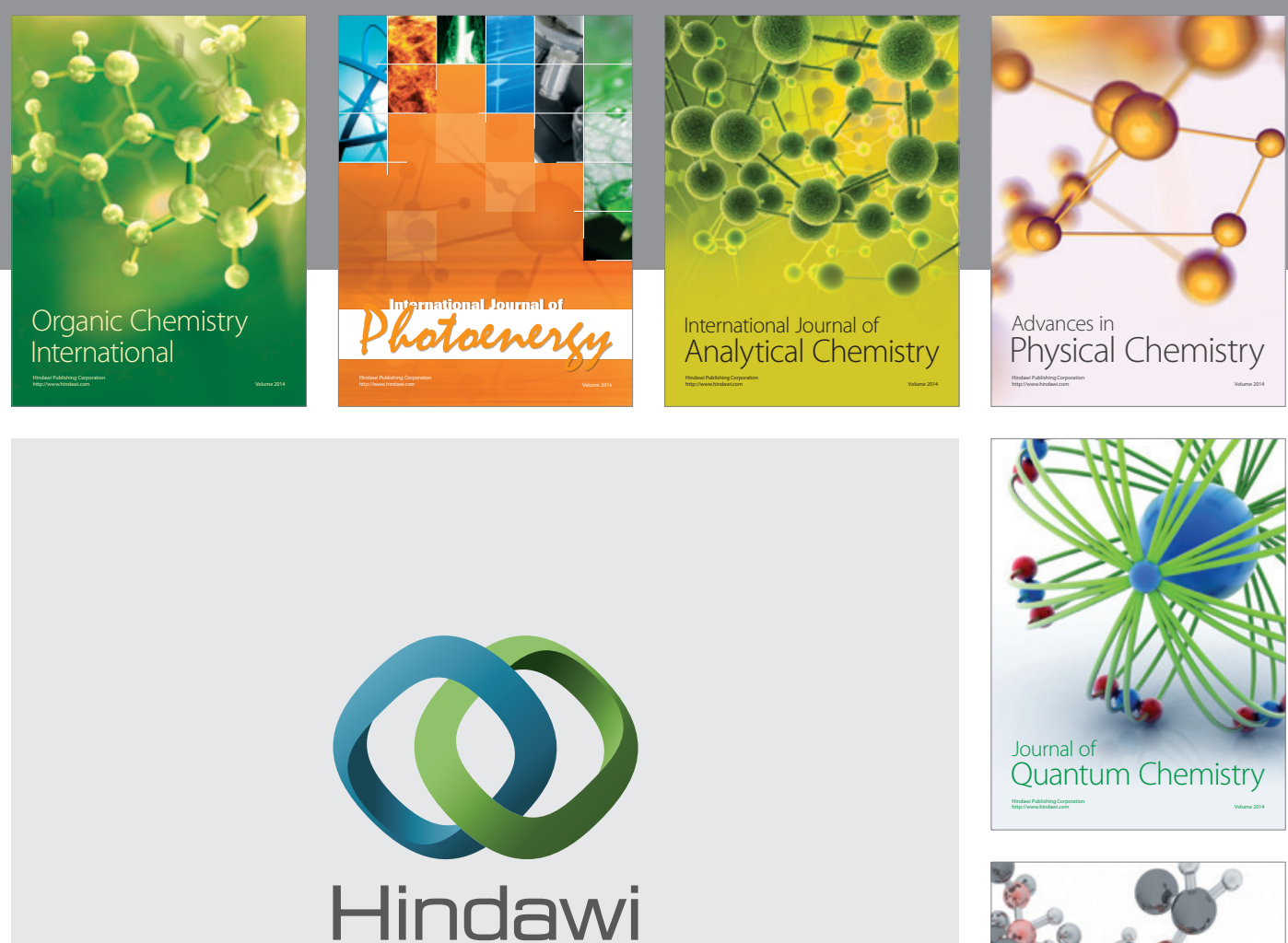

Submit your manuscripts at

http://www.hindawi.com

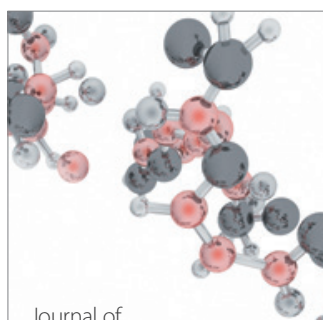

Analytical Methods

in Chemistry

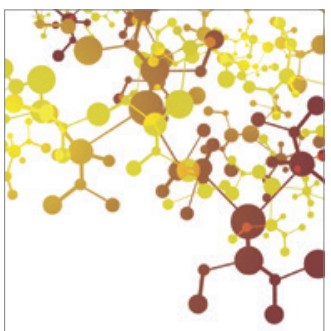

Journal of

Applied Chemistry

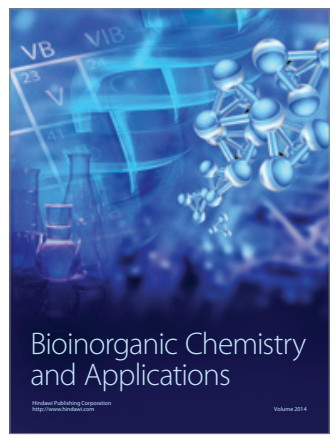

Inorganic Chemistry
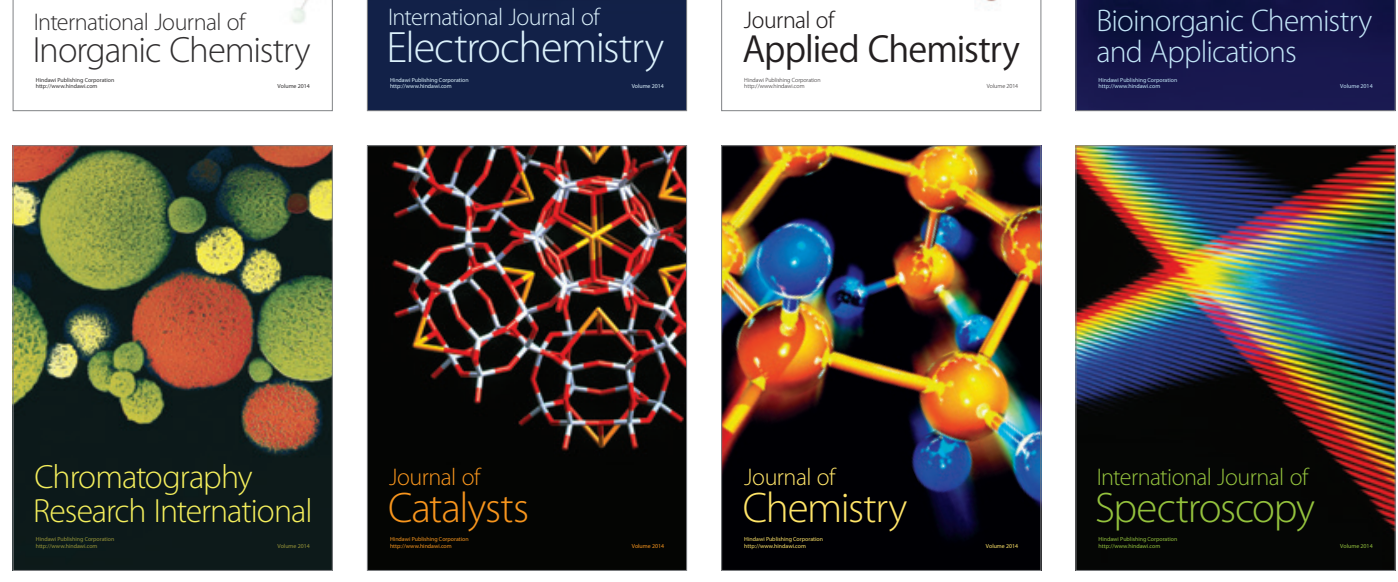